\title{
Binding of sodium aurothiomalate to human serum albumin in vitro at physiological conditions
}

\author{
SUSANNE MØLLER PEDERSEN \\ From the Department of Clinical Chemistry, Odense University Hospital, Odense; and the Rheumatism Unit, \\ Aarhus University, Aarhus, Denmark
}

SUMMARY The binding of aurothiomalate to human serum albumin was studied by equilibrium

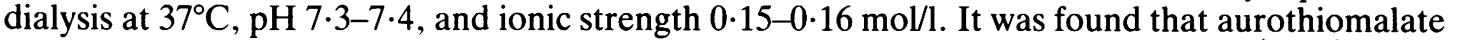
was bound to albumin at one site with an apparent association constant $\mathrm{K}_{1}=3 \cdot 0 \times 10^{4} \mathrm{M}^{-1}$ and at three or more sites with the sum of association constants of the order of $10^{3} \mathrm{M}^{-1}$. Valuable information of the aurothiomalate-albumin interaction was deduced from the observed changes $\vec{N}$ of $\mathrm{pH}$ of the albumin solutions during dialysis. A conceivable binding mechanism consistent with $\cong$ the results might be that aurothiomalate binds as $\mathrm{Au}^{+}$to the high affinity binding site by exchanging a $\mathrm{H}^{+}$and that this site might be the sulphydryl group in cysteine ${ }_{34}$; and that $\mathbb{D}$ aurothiomalate binds as monomeric anions to the lower affinity binding sites.

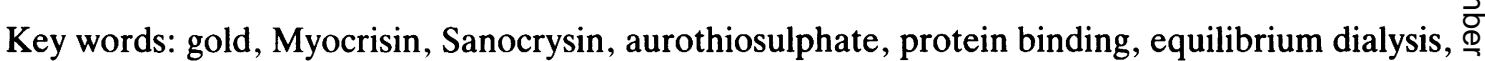
binding mechanism, gold therapy.

Gold salts in the form of thio complexes have been used successfully in the treatment of rheumatoid arthritis for more than 50 years. Important aspects of their pharmacokinetics and mode of action, however, are still unknown. Several reports indicate a lack of correlation between the total concentration of gold in plasma and therapeutic and toxic effects. $^{1-8}$ In vivo and in vitro experiments suggest that most of the gold in the circulation is bound to albumin..$^{2-10}$ Since it is conceivable that the clinical effects might be correlated with the non-protein bound gold concentration this value might be a more useful parameter for monitoring patients during chrysotherapy. In order to calculate the free gold concentration in plasma (or serum) reliable values for the apparent association constants are needed.

Very little quantitative information concerning the binding of gold compounds to human serum albumin is available. Danpure found that aurothiomalate was bound to human albumin in vitro at a single site, of which 0.7 sites had an apparent association constant of $1.5 \times 10^{6} \mathrm{M}^{-1}$ and 0.3 sites had an apparent association constant of $1.1 \times 10^{5}$ $\mathbf{M}^{-1}$. ${ }^{10}$ Mason found that aurothiomalate was bound to albumin in vitro at a single site with an association

Accepted for publication 19 February 1986.

Correspondence to Dr Susanne Møllc, Pedersen, Klinisk kemisk afdeling, Odense Sygehus, DK-5000 Odense C, Denmark. constant $\mathrm{K}_{1}=6 \cdot 10 \times 10^{3} \mathrm{M}^{-1}$ and at 6.6 sites with $\mathrm{K}_{2}=2.35 \times 10^{2} \mathrm{M}^{-1},{ }^{11}$ and Pedersen found that aurothiosulphate was bound to human albumin in vitro at one site with an association constant $\mathrm{K}_{1}=3.0 \times 10^{4} \mathrm{M}^{-1}$ and at three or more sites with the $\stackrel{\mathbb{Q}}{\stackrel{\mathbb{Q}}{\circ}}$ sum of the association constants equal to $1.6 \times 10^{3} \overrightarrow{\vec{B}}$ $\mathrm{M}^{-1} \cdot{ }^{12}$ These results differ considerably from each 3 other possibly owing to the different techniques? employed or to the different analysis of the binding? data, or for both these reasons.

Reliable association constants are of clinical importance as gold compounds on empirical base $\frac{0}{3}$ are administered in equivalent doses with respect to gold content. In the study presented here the $O$ binding of sodium aurothiomalate to human serum albumin was investigated in vitro at physiological 음 conditions with respect to albumin concentration, $>$ $\mathrm{pH}$, temperature, and ionic strength. As it was found that aurothiomalate was strongly bound to the $\bar{N}$ dialysis membrane an equilibrium dialysis system $\%$ was used, with the great advantage that partial $N$ adsorption to the membrane does not influence the results. ${ }^{13}$ The experimental conditions and the analysis of the binding data are identical to those previously described for the binding of aurothio- $\frac{\widetilde{D}}{\mathrm{D}}$ sulphate to human albumin. ${ }^{12}$ It is, therefore, $\stackrel{\oplus}{+}$ possible to compare the results obtained for the two gold compounds. 


\section{Materials and methods}

\section{A T E R I A L S}

The albumin preparation used was purified, lyophilised human albumin (Behringwerke AG, Marburg, West Germany). The albumin preparation fulfilled the criteria for purity specified by Hobbs et al. ${ }^{14}$ Polyacrylamide gradient gel (PAA 4/30, Pharmacia, Uppsala, Sweden) electrophoresis of a $0.5 \%$ albumin solution showed only one distinct band of monomer albumin and one very faint band due to dimer albumin. Crossed immunoelectrophoresis ${ }^{15}$ performed against rabbit antiserum (Dako, Copenhagen, Denmark) showed that no proteins other than albumin were detectable. The sodium aurothiomalate $\left(\mathrm{AuSH}_{3} \mathrm{C}_{4} \mathrm{O}_{4} \mathrm{Na}_{2}\right)$ was purchased from Rhône-Poulenc Pharma Norden, Birkerød, Denmark. The visking seamless cellophane tubing (8/32 inch, Union Carbide Corporation, Chicago) used for dialysis was washed and prepared as described by Pedersen. ${ }^{16}$ All initial solutions of albumin, aurothiomalate, and blanks were unbuffered solutions prepared in distilled, sterile water containing $0 \cdot 15 \mathrm{M} \mathrm{NaCl}$ and with $\mathrm{pH}$ adjusted to 7.50 in order to obtain $\mathrm{pH} \simeq 7.4$ at equilibrium (see below).

\section{EQUILIBRIUM DIALYSIS}

The binding of aurothiomalate to human albumin in unbuffered solutions at $37^{\circ} \mathrm{C}, \mathrm{pH} 7 \cdot 3-7 \cdot 4$, and ionic strength $0 \cdot 15-0 \cdot 16 \mathrm{~mol} / \mathrm{l}$ was studied in an equilibrium dialysis system containing initially $1 \mathrm{ml}$ albumin solution on the inside and $3 \mathrm{ml}$ aurothiomalate solution on the outside of the membrane with a range of concentrations of total sodium aurothiomalate from $102 \mu \mathrm{mol} / \mathrm{l}$ to $2051 \mu \mathrm{mol} / \mathrm{l}$.

Control tubes, with aurothiomalate solution on the outside and blanks on the inside of the dialysis membrane, were set up at two different aurothiomalate concentrations in duplicate to establish that equilibrium was reached at the end of the experiment. During dialysis the samples were vertically rotated in order to obtain equilibrium within 48 hours. After equilibrium was reached the concentration of albumin was measured inside and the $\mathrm{pH}$ and concentrations of gold and sodium were measured on both sides of the dialysis membrane.

\section{ONIC STRENGTH}

According to general practice the contribution of albumin to ionic strength was ignored and it was found that, in the concentration range of sodium aurothiomalate used, the contribution of aurothiomalate to the ionic strength in each albumin solution could be neglected.

\section{$\mathrm{pH}$}

During dialysis it was found that $\mathrm{pH}$ decreased by $0 \cdot 15-0 \cdot 30 \mathrm{pH}$ units in the albumin containing solutions and by approximately $0.20 \mathrm{pH}$ units in the solutions free of macro-ions. The changes in $\mathrm{pH}$ during dialysis are illustrated in Table 1 . In order to obtain $\mathrm{pH}$ equal to $7 \cdot 3-7 \cdot 4$ at equilibrium the $\mathrm{pH}$ was adjusted before dialysis to $\mathbf{7 \cdot 5 0 - 7 . 5 5}$ in each initial solution.

\section{MEASUREMENTS}

For albumin determinations a quantitative electroimmunoassay technique was used according to the principles of Laurell ${ }^{15}$ (albumin standard: standard human serum, Behringwerke AG). The gold concentrations were determined with a flameless atomic absorption spectrophotometer (Beckman model 485 fitted with a Masmann cuvette model 1268) as described by Pedersen and Graabaek. ${ }^{18}$ All $\mathrm{pH}$ measurements were performed at $37^{\circ} \mathrm{C}$ with a Radiometer $\mathrm{pH}$ meter PHM 72 supplied with the electrode system BMS 2 MK 2 blood microsystem.

\section{Results}

Fig. 1 shows the experimental data for the binding of sodium aurothiomalate to human serum albumin at neutral $\mathrm{pH}$; the temperature was $37^{\circ} \mathrm{C}$ and the ionic strength $0 \cdot 15-0 \cdot 16 \mathrm{~mol} / 1$ in all solutions. The binding data are plotted as $\bar{v}$ versus $\log C$, where $\bar{v}$ is the average number of gold atoms bound (in one form or another) per albumin molecule and $C$ is the equilibrium concentration of unbound gold. In the present study the total concentration of gold was determined on both sides of the dialysis membrane when equilibrium was reached. The measurement on the inside yields the sum of protein bound and

Table 1 Changes in $p H$ during dialysis

\begin{tabular}{lll}
\hline$v^{*}$ & $-\Delta p H^{+}$ & $-\Delta p H^{\prime}+$ \\
\hline $0 \cdot 2$ & $0 \cdot 23$ & $0 \cdot 19$ \\
$0 \cdot 3$ & $0 \cdot 28$ & $0 \cdot 22$ \\
$0 \cdot 4$ & $0 \cdot 28$ & $0 \cdot 21$ \\
$0 \cdot 5$ & $0 \cdot 26$ & $0 \cdot 21$ \\
$0 \cdot 6$ & $0 \cdot 26$ & $0 \cdot 21$ \\
$0 \cdot 8$ & $0 \cdot 24$ & $0 \cdot 20$ \\
$1 \cdot 1$ & $0 \cdot 21$ & $0 \cdot 20$ \\
$1 \cdot 6$ & $0 \cdot 20$ & $0 \cdot 20$ \\
$2 \cdot 0$ & $0 \cdot 16$ & $0 \cdot 20$
\end{tabular}

The ionic strength was $0 \cdot 15 \mathrm{~mol} / \mathrm{l}$, the temperature $37^{\circ} \mathrm{C}$, and the albumin concentration $0.51 \mathrm{mmol} / \mathrm{l}$.

*The average number of gold atoms bound per albumin molecule. †The mean value of the observed changes in $\mathrm{pH}$ of the albumin solutions during dialysis from the three independent experiments. The primed symbol indicates the side of the membrane free of macro-ions. 


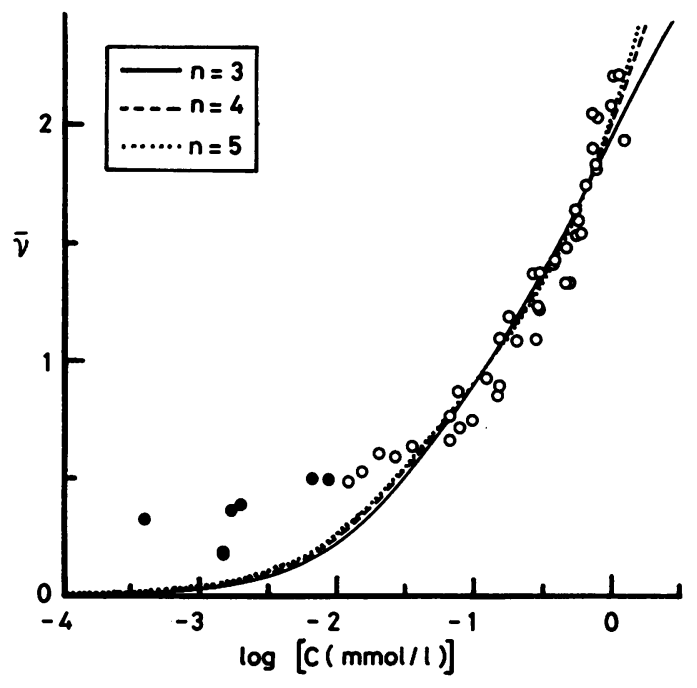

Fig. 1 Binding of aurothiomalate to human albumin at $37^{\circ} \mathrm{C}, \mathrm{pH} 7.3-7.4$ and ionic strength $0.15-0.16 \mathrm{molll}$; the experimental points represent three strictly independent sets of experiments; the lines represent the best fit to equation (1) for the indicated values of $n$. The points indicated by $(\Theta)$ are not included in the analysis, $c f$. the text.

non-protein bound gold. That of the outside is a measure of non-protein bound gold. The protein bound gold was then calculated as the difference between total gold and non-protein bound gold; and $\bar{v}$ evaluated by the equation $\bar{v}=$ [albumin bound gold]/[albumin]. The molecular weight of albumin was assumed to be 67000 throughout.

Although the gold or gold complex are probably present as ions, no correction for the Donnan effect was made as the concentration of added salt $(0 \cdot 15 \mathrm{M}$ $\mathrm{NaCl}$ ) was sufficiently high to suppress this; e.g., the observed distribution factor for $\mathrm{Na}^{+}$across the semipermeable membrane $(\mathrm{Na}) /\left(\mathrm{Na}^{\prime}\right)$ was 1.01 , where the primed symbol indicates the side of the membrane free from macro-ions.

The lines in Fig. 1 are the best fit to the equation:

$$
\bar{v}=\sum_{i=1}^{n} \frac{K_{i} C}{1+K_{i} \mathrm{C}}
$$

using a non-linear least square curve fitting procedure. The summation is over all $n$ sites of the albumin molecule, and $\mathrm{K}_{\mathrm{i}}$ is the association constant for site i. Equation (1) implies that there is no interaction between the sites. The maximum number of binding sites, $\mathrm{n}$, is not known and was, therefore, varied in the calculations. The binding

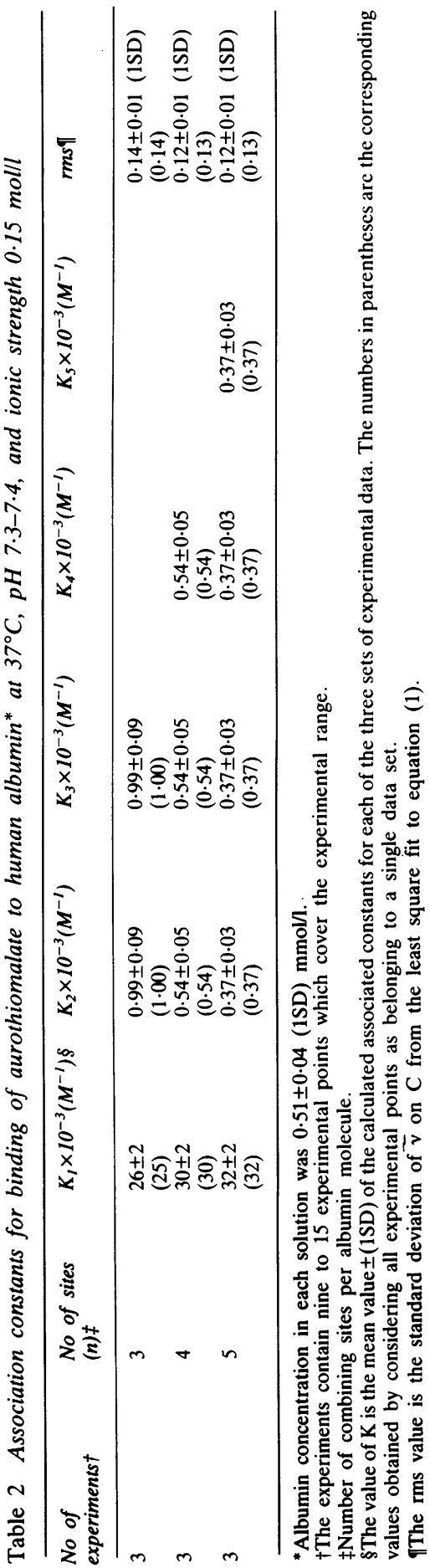


data $\{\bar{v}, C\}$ for small gold concentrations (indicated by (O) in the figure) could not be fitted to the calculated lines and were therefore not included in the analysis. The reason for this interesting discrepancy at small gold concentrations is under further investigation. If these binding data were included, however, the association constant $\mathrm{K}_{1}$ was increased by $60 \%$ and the other constants remained virtually unchanged.

Table 2 summarises the binding results obtained for the three independent sets of experimental data considered separately or as a single set. The results show that there is a single high affinity binding site with an association constant $\mathrm{K}_{1}=3 \times 10^{4} \mathrm{M}^{-\mathrm{P}}$ and three or more lower affinity binding sites with association constants of the order of $10^{3} \mathrm{M}^{-1}$. The best fit to equation (1) was significantly improved by increasing $\mathrm{n}$ from three to four, but only minor changes of the rms values resulted when $n$ was increased beyond four. The possibility that more than four binding sites exist cannot be excluded. The association constants for sites two to four were identical. The results did not depend on whether the three sets of experimental data were analysed separately or as a single data set.

\section{Discussion}

In the present investigation it was found that aurothiomalate was bound to human serum albumin in vitro at one site with an association constant $\mathrm{K}_{1}=3 \times 10^{4} \mathrm{M}^{-1}$ and at three or more sites with the sum of association constants of the order of $10^{3}$ $\mathbf{M}^{-1}$.

In an attempt to identify the combining sites and understand the mode of action of the compound the values of the association constants and the number of combining sites might be important. For clinical purposes, however, it is the determination of the equilibrium constant $k_{1}$, equal to the sum of the calculated association constants $\left(\sum_{i=1}^{n} K_{i}\right)$, which is of interest. The range of $k_{1}$ values is $2.7 \times 10^{4} \mathrm{M}^{-1}$ to $3.6 \times 10^{4} \mathrm{M}^{-1}$, with $\mathrm{n}=3$ to $\mathrm{n}=8$. These values of $\mathrm{k}_{1}$ correspond to $94-96 \%$ binding of gold to human serum albumin under physiological conditions.

Mason's results for the binding of aurothiomalate to human albumin ${ }^{11}$ give a value of $k_{1}$ which corresponds to $80-82 \%$ binding of gold to human serum albumin. The lower degree of binding of aurothiomalate might be due to problems of drugmembrane binding in the ultrafiltration method used in this study. ${ }^{11}$ The association constants obtained by Danpure may not be reliable owing to an inadequate analysis of the binding data. ${ }^{10}$

The degree of gold binding found in this study agrees well with the results obtained by Mas- carenhas et al, ${ }^{2}$ van de Stadt and Abbo-Tilstra, ${ }^{\gamma}$ and Ward et al, ${ }^{9}$ who measured the plasma gold distribution during chrysotherapy in vivo in patients with rheumatoid arthritis. Using different techniques they found that $85-95 \%$ of the gold was bound to albumin.

During dialysis a decrease in $\mathrm{pH}$ in all the solutions was observed (Table 1). The $\mathrm{pH}$ changes in the albumin containing solution, $\Delta \mathrm{pH}$, and in the solution free of macro-ions, $\Delta \mathrm{pH}^{\prime}$, might give some valuable information of the aurothiomalate-albumin interaction. From Table 1 it is seen that $\Delta \mathrm{pH}$ decreases from -0.28 to -0.16 with increasing $\bar{v}$, whereas $\Delta \mathrm{pH}^{\prime} \simeq-0 \cdot 20$ is independent of $\bar{v}$. The observed change in $\mathrm{pH}$ in the albumin containing solution might be due to the binding of gold or gold complex to the albumin molecule changing its attraction to hydrogen ions. For $\bar{v}<1$ the observed decrease of $\mathrm{pH}$ might be explained by assuming that the gold complex binds to albumin as $\mathrm{Au}^{+}$by exchanging a $\mathrm{H}^{+}$and releasing its free thiomalate. For $\bar{v} \geqslant 1$ the change in $\mathrm{pH}$ might be explained by assuming that a negatively charged gold complex binds to albumin. The change of charge of the albumin molecule due to combination with anions increases its attraction to hydrogen ions, thus increasing the $\mathrm{pH}$ in the albumin containing solutions. The binding mechanism suggested in the present study agrees with that suggested for the binding of aurothiosulphate to human albumin. ${ }^{19} 20$

Although aurothiomalate and aurothiosulphate are respectively organic and inorganic compounds, the similar values for the association contants of the two compounds also support the hypothesis that the same binding mechanism applies. Assuming $n=4$ identical values of $K_{1}$ are obtained, suggesting that it is the sulphydryl group ( $\mathrm{SH}$ ) of Cys(34) which is the high affinity binding site. ${ }^{12} 1920$ The SH content of the albumin preparation used was $0.30 \mathrm{~mol} / \mathrm{mol}$ albumin determined by the method of Janatová $e t$ $a l .^{21}$ The importance of the free sulphydryl content, however, is not clear, ${ }^{22}$ and therefore assumptions about the SH content have not been included in the present analysis. The association constant for the lower affinity binding sites in aurothiomalate solution is twice that obtained for aurothiosulphate solution. ${ }^{12} 19$ This can be explained using the Linderstrøm-Lang equation with the relevant parameter values ${ }^{19}$ by assuming that the charge of the binding complex is one larger in the aurothiomalate solution than in the aurothiosulphate solution. It has been shown that aurothiosulphate is bound as the gold complex, $\mathrm{Au}\left(\mathrm{S}_{2} \mathrm{O}_{3}\right)_{2}^{3-}$, to the lower affinity binding site. ${ }^{19}{ }^{20}$ Consequently, the present results indicate a charge of -2 of the binding complex in the aurothiomalate solution. This is consistent with 
binding of monomer aurothiomalate anions, $\mathrm{AuSH}_{3} \mathrm{C}_{4} \mathrm{O}_{4}^{2-}$, to the lower affinity binding sites.

There is evidence that aurothiomalate exists as a polymer in aqueous solutions, ${ }^{23-25}$ though its precise chemical nature has not yet been established. ${ }^{26}$ The degree of polymeric association is known to be strongly dependent on ionic strength, aurothiomalate concentration, and $\mathrm{pH}$ of the solution and decreases with decreasing concentration and ionic strength. ${ }^{25}$ In the present experiments both the ionic strength and the aurothiomalate concentrations are much smaller than those used in the structural investigations mentioned above and, therefore, the possibility that monomer aurothiomalate is the main constituent in the investigated solutions cannot be excluded. The possible presence of polymeric forms in the solution is interesting and noteworthy and is also consistent with the above analysis if these polymers are broken down to monomers upon binding.

The suggested binding mechanism supports the finding of free thiomalate in plasma and urine of patients receiving sodium aurothiomalate. ${ }^{27-29}$ Due to these findings and the structural resemblance of penicillamine and aurothiomalate ligands it has been suggested that thiomalate might be the active metabolite in 'gold' therapy. ${ }^{27-29}$ From the essentially identical binding results obtained for the binding of sodium aurothiosulphate to human serum albumin $^{12}$ one could equally suggest that thiosulphate might be the active metabolite in aurothiosulphate therapy, as no differences in clinical effects of the two compounds have ever been reported. The two compounds have no structural resemblance except that both are drugs containing thiol. If the above mentioned suggestions are correct this opens some very interesting perspectives for future treatment of rheumatoid arthritis; and for the same reasons as mentioned for thiomalate $e^{29}$ a controlled trial of thiosulphate is clearly indicated.

Although attention is now being paid to the possible antirheumatic activity of the thiomalate and thiosulphate ligands, it should not be forgotten that gold compounds have been popular drugs for the treatment of many diseases for centuries. Thiols were first introduced as $\mathrm{Au}(\mathrm{I})$ stabilising ligands between 1913 and 1927, a period of intense searching for $\mathrm{Au}(\mathrm{I})$ compounds of lower toxicity. It is still likely that it is the free concentration of the gold thio complexes in the tissue fluids which determines pharmacological activity. As these compounds are strongly bound to human serum albumin, minor changes in the binding affinity or capacity might give rise to a major change in the free 'gold' concentration, which could induce unwanted clinical effects. The lack of correlation between total gold concen- tration in plasma and clinical effects is easily understood. There might, however, be a correlation between the free gold concentration in plasma and therapeutic and toxic effects. This remains to be $\vec{s}$ evaluated. For practical clinical purposes it is not $\bar{O}$ possible to determine the in vivo free 'gold' $\frac{C}{\sigma}$ concentration. Knowing the association constants, $\overline{\frac{\rho}{}}$ the albumin concentration, and the total concen- $\mathbb{D}$ tration of 'gold' in plasma or serum, it is possible to calculate the free 'gold' concentration. The present results show that under physiological conditions no significant difference in the binding of aurothiomalate and aurothiosulphate to human serum albumin could be demonstrated.

The author is greatly indebted to Mrs Inge Bihlet for skilful technical assistance.

\section{References}

1 Gerber R C, Paulus H E, Bluestone R, Pearson C M. Clinical $\stackrel{\bigcirc}{\supset}$ response and serum gold levels in chrysotherapy. Ann Rheum Dis 1972; 31: 308-10.

2 Mascarenhas B R, Granda J L, Freyberg R H. Gold metabol- $\frac{\mathbb{D}}{\mathrm{C}}$ ism in patients with rheumatoid arthritis treated with gold $\mathbb{D}$ compounds-reinvestigated. Arthritis Rheum 1972; 15: 391-402.

3 Rubinstein H M, Dietz A A. Serum gold. II. Levels in rheumatoid arthritis. Ann Rheum Dis 1973; 32: 128-32.

4 Jessop J D, Johns R G S. Serum gold determinations in patients $\vec{c}$ with rheumatoid arthritis receiving sodium aurothiomalate Ann Rheum Dis 1973; 32: 228-32.

5 Gottlieb N L, Smith P M, Smith E M. Pharmacodynamics ${ }^{197} \mathrm{Au}$ and ${ }^{195} \mathrm{Au}$ labelled aurothiomalate in blood: correlation with course of RA, gold toxicity and gold excretion. Arthritis Rheum 1974; 17: 171-83.

6 Sharp J T, Lidsky M D, Duffy J, et al. Comparison of two dosage schedules of gold salts in the treatment of rheumatoid $\mathbb{D}$ arthritis. Arthritis Rheum 1977; 20: 1179-87.

7 Furst D E, Levine S, Srinivasan R, Metzger A L, Bangert R, Paulus $\mathrm{H}$ E. A double-blind trial of high versus conventional dosages of gold salts for rheumatoid arthritis. Arthritis Rheum 1977; 20: 1473-80.

8 van de Stadt R J, Abbo-Tilstra B. Gold binding to blood cells and serum proteins during chrysotherapy. Ann Rheum Dis 1980; 39: 31-6.

9 Ward R J, Danpure C J, Fyte D A. Determination of gold in plasma and plasma fractions by atomic absorption spectrometry and by neutron activation analysis. Clin Chim Acta 1977; 81: 87-97.

10 Danpure C J. Interaction of aurothiomalate with human serum albumin in vitro. Biochem Soc Trans 1976; 4: 161-3.

11 Mason $\mathrm{R} \mathrm{W}$. The binding of aurothiomalate to plasma proteins in vitro. Pharmacology 1977; 15: 536-44.

12 Pedersen S M. The binding of gold to human albumin in vitro. Intrinsic association constants at physiological conditions. O Biochem Pharmacol 1981; 30: 3249-52.

13 Eisen H N. Equilibrium dialysis for measurement of antibody- $\mathrm{N}$ hapten affinities. Methods Med Res 1964; 10: 106-14.

14 Hobbs J R, Harboe N, Alper C, Johansson B G, Peters T. Provisional recommendation (1978) on specification for human serum albumin standard. Clin Chim Acta 1979; 98: 175-8F. Co

15 Weeke B. Crossed immunoelectrophoresis. Scand J Immunol 1973; (suppl 1): 47-56.

16 Pedersen K O. Multiple small sample ultrafiltration without special apparatus. Scand J Clin Lab Invest 1971; 28: 57-9.

17 Laurell C-B. Quantitative estimation of proteins by electro- 
phoresis in agarose gel containing antibodies. Anal Biochem 1966; 15: 45-52.

18 Pedersen S M, Graabaek P M. A unified method for the determination of gold in biological fluids by flameless atomic absorption spectroscopy. Scand J Clin Lab Invest 1977; 37: 91-4.

19 Pedersen S M. The effect of $\mathrm{pH}$ on the binding of sodium aurothiosulphate to human serum albumin. A possible binding mechanism. Biochem Pharmacol 1983; 32: 2485-8.

20 Pedersen S M. Influence of ionic strength on the binding of sodium aurothiosulphate to human serum albumin. Biochem Pharmacol 1985; 34: 4319-23.

21 Janatová J, Fuller J K, Hunter M J. The heterogeneity of bovine albumin with respect to sulphydryl and dimer content. $J$ Biol Chem 1968; 243: 3612-22.

22 Katchalski E, Benjamin G S, Gross V J. The availability of the disulphide bonds of human and bovine serum albumin and of bovine $\gamma$-globulin to reduction by thioglycolic acid. J Am Chem Soc 1957; 79: 4096-9.

23 Sadler P J. The biological chemistry of gold: a metallo-drug and heavy-atom label with variable valency. Structure Bonding 1976: 29: 171-215.

24 Sadler P J. The comparative evaluation of the physical and chemical properties of gold compounds. $J$ Rheumatol 1982; 9 (suppl 8): 71-8.

25 Isab A A, Sadler P J. Hydrogen-1 and carbon-13 nuclear magnetic resonance studies of gold(1) thiomalate ('Myocrisin') in aqueous solution: dependence of the solution structure on $\mathrm{pH}$ and ionic strength. J Chem Soc (Dalton) 1981: 1657-63.

26 Harvey D A, Kean W F, Lock C J L, Singal D. Sodium aurothiomalate is a mixture. Lancet 1984 ; i: $470-1$.

27 Jellum E, Munthe E. Fate of the thiomalate part after intramuscular administration of aurothiomalate in rheumatoid arthritis. Ann Rheum Dis 1982; 41: 431-2.

28 Rudge S R, Perrett D, Swannell A J. Free thiomalate in plasma and urine of patients receiving sodium aurothiomalate. Ann Rheum Dis 1984; 43: 66-9.

29 Rudge S R, Perrett D, Swannell A J, Drury P L. Circulating thiomalate after administration of disodium aurothiomalate. Impurity or active metabolite. J Rheumatol 1984; 11: 150-2. 